\title{
Clinical Application Value of Combined Detection of CG and TBA in Differential Diagnosis of Hepatobiliary System Diseases
}

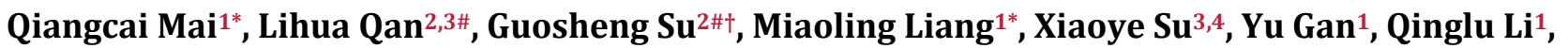
Can Long ${ }^{1}$, Huayan Zhu' ${ }^{1}$, Qiaocui Jiang ${ }^{1}$, Yanling Zheng ${ }^{1}$, Jieli He ${ }^{1}$, Tianwu Liang ${ }^{1}$, Jing Li ${ }^{1}$, Buqing Su ${ }^{5}$

${ }^{1}$ Department of Gastroenterology/Laboratory, Guigang Orthopedic Hospital of Integrated Traditional Chinese and Western Medicine, Guigang, China; ${ }^{2}$ Department of Internal Medicine/Laboratory Department of the People's Hospital of Guangxi ASEAN Economic and Technological Development Zone, Nanning Tenth People's Hospital, Nanning, China; ${ }^{3}$ Department of Nursing, Graduate School, Eternal University of the Philippines, Las Pinas, Philippines; ${ }^{4}$ Department of Critical Medicine, Fuzhou Second People's Hospital, Fuzhou, China; ${ }^{5}$ Nursing Department of Liaoning Hospital Vocational College, Shenyang, China

Correspondence to: Guosheng Su, 563449581@qq.com

Keywords: Cholyglycine, Total Bile Acid, Hepatobiliary Diseases, Primary Liver Cancer, The Differential Diagnosis

Received: January 24, $2022 \quad$ Accepted: February 25, $2022 \quad$ Published: February 28, 2022

Copyright $\odot 2022$ by author(s) and Scientific Research Publishing Inc.

This work is licensed under the Creative Commons Attribution International License (CC BY 4.0).

http://creativecommons.org/licenses/by/4.0/

\section{(c) (i) Open Access}

\section{ABSTRACT}

Objective: To understand the clinical value of combined detection of cholyglycine CG and TBA in differential diagnosis of hepatobiliary diseases. Methods: Serum samples from 50 healthy people were collected as healthy control group. According to the latest disease diagnosis and treatment plan, 58 cases of $\mathrm{HBV}$ asymptomatic carrier group, 17 cases of viral hepatitis group, 49 cases of cirrhosis group, 50 cases of primary liver cancer group and 50 cases of other hepatobiliary diseases groups were collected respectively. The concentration levels of cholyglycine and total bile acid in each group were detected, and the differences among each group were compared. Results: By statistical analysis, serum CG concentration in viral hepatitis group, cirrhosis group, primary liver cancer group and other hepatobiliary diseases group was significantly higher than that in asymptomatic HEPATITIS B carriers and healthy control group, the differences were statistically significant $(p<0.05)$. While, the serum CG concentration of asymptomatic hepatitis B virus carrier group was similar to that of healthy control group, the difference was not statistically significant $(p>0.05)$. There was

\footnotetext{
${ }^{*}$ Co-first author.

${ }^{\#}$ Co-corresponding author.

${ }^{\dagger}$ Corresponding author.
} 
no significant difference in CG concentration among viral hepatitis group, liver cirrhosis group, primary liver cancer group and other hepatobiliary diseases group ( $p>0.05)$. The serum TBA levels of asymptomatic carriers, viral hepatitis group, cirrhosis group, primary liver cancer group and other hepatobiliary system diseases group were significantly higher than those of healthy control group, the difference was statistically significant, $\mathrm{p}<0.05$. There was no significant difference in TBA concentration among the asymptomatic carriers of hepatitis B virus, viral hepatitis group, liver cirrhosis group, primary liver cancer group and other hepatobiliary system disease groups, all $\mathrm{p}>0.05$. Conclusion: Serum CG expression can not only detect liver lesions, but also distinguish different liver lesions. The positive rate of CG combined with TBA detection in patients with hepatobiliary diseases is significantly higher than that of single CG index detection. CG combined with TBA detection can significantly improve the sensitivity and accuracy of the diagnosis of hepatobiliary diseases, which is worthy of popularization and application.

\section{RESEARCH BACKGROUND}

Cholyglycine (CG) is secreted by liver cells and is the main bile acid in serum during the third trimester of pregnancy. Similar to other bile acids, it is metabolized in the body through the "entero-hepatic circulation", and the serum CG level of normal people is very low. When hepatocytes are damaged or cholestasis occurs, the disorder of Cholyglycinemetabolism is caused, or when hepatocytes are damaged or necrotic, a large amount of glycine enters the peripheral blood, which can increase the content of glycocholic acid in serum [1-3]. Therefore, it can be considered as a sensitive index to indicate the impairment of liver function. In order to understand the clinical application value of the combined detection of cholyglycine CG and TBA in the differential diagnosis of hepatobiliary diseases, this topic will collect the serum of patients with hepatobiliary diseases and healthy controls to detect the serum glycine level alone or jointly, and explore the application value of glycocholic acid in hepatobiliary diseases through statistical analysis.

\section{DATA AND METHODS}

\subsection{Research Objects}

Healthy control group: Serum samples from 50 healthy people were collected. Disease group: According to the latest disease diagnosis and treatment plan, 58 cases of hepatitis B virus asymptomatic carrier group, 17 cases of viral hepatitis group, 49 cases of liver cirrhosis group, 50 cases of primary liver cancer group and 50 cases of other hepatobiliary system disease groups were collected. All cases entering the disease group were required not to be complicated with other major diseases. The collected serum samples were free of lipid blood and hemolysis; Collect gender, age, diagnosis and other information and other test results. All enrolled patients obtained their own signed consent and were discussed and approved by the medical ethics committee of the unit before implementation.

\subsection{Research Methods}

The collected serum samples were numbered, centrifuged to separate the serum, and the serum that was not detected in time was packaged and stored in a $-80^{\circ} \mathrm{C}$ refrigerator. The levels of cholyglycine and total bile acid were detected in serum groups. The reagents, calibrators and quality control materials used in the detection process were all provided by Jiuqiang Biological, and the same biochemical analyzer was used for analysis in the detection process. The detection process ensured the freshness of the samples, and excluded the influence of excessive sample storage time or repeated freezing and thawing on the detection results. 


\subsection{Data Processing and Statistical Analysis}

SPSS 24.0 was used for data processing and statistical analysis, and $\mathrm{p}<0.05$ was considered to be statistically significant.

\section{RESULTS}

A total of 274 specimens were collected. Through statistical analysis, it was found that the serum CG levels in the viral hepatitis group, liver cirrhosis group, primary liver cancer group and other hepatobiliary disease groups were significantly higher than those in asymptomatic carriers of hepatitis B virus and healthy controls, and the differences were statistically significant, $\mathrm{p}<0.05$. While, the serum CG concentration in asymptomatic carriers of hepatitis $\mathrm{B}$ virus was similar to that in healthy controls, and there was no significant difference between them ( $p>0.05$ ). There was no significant difference in the concentration of CG among viral hepatitis group, liver cirrhosis group, primary liver cancer group and other hepatobiliary diseases group, $\mathrm{p}>0.05$. Serum TBA levels in asymptomatic carriers of hepatitis B virus, viral hepatitis group, liver cirrhosis group, primary liver cancer group and other hepatobiliary disease groups were significantly higher than those in the healthy control group, the differences were all statistically significant, $\mathrm{p}<0.05$. There was no significant difference in the levels of TBA concentration among the asymptomatic carriers of hepatitis B virus, viral hepatitis group, liver cirrhosis group, primary liver cancer group and other hepatobiliary system disease groups, all $\mathrm{p}>0.05$. The specific results are shown in Table 1 .

Annotations: healthy control group, hepatitis B virus asymptomatic carrier group, viral hepatitis group, liver cirrhosis group, primary liver cancer group, and other hepatobiliary diseases group were replaced by (1), (2), (3), (4), (5), and (6) respectively. The comparison of each group is shown in Table 2.

\section{DISCUSSION}

It is reported [4-9] that liver diseases led by liver cancer are the common cause of death among Chinese residents. Primary liver cancer and cirrhosis are often developed from viral hepatitis, alcoholic hepatitis and non-alcoholic steatohepatitis. Moreover, due to the increase of obesity level and diabetes prevalence year by year, some models predict that the prevalence of non-alcoholic fatty liver disease will increase steadily in China before 2030, and the incidence of advanced liver disease and liver disease mortality will more than double. At present, the biochemical indicators used in clinical hepatobiliary diseases are mainly aspartate aminotransferase (AST), alanine aminotransferase (ALT) and $\gamma$-glutamyl transpeptidase ( $\gamma$-glutamyl transpeptidase), etc., but there are limitations such as poor specificity in suggesting liver damage. With the continuous development of testing technology and the emergence of new testing items, it is imminent to find new indicators related to hepatobiliary diseases.

Table 1. Concentration results of serum CG and TBA in various hepatobiliary diseases.

\begin{tabular}{cccc}
\hline Group & Number of cases & CG $(\mathrm{mg} / \mathrm{L})$ & TBA $(\mu \mathrm{mol} / \mathrm{L})$ \\
\hline $\begin{array}{c}\text { Healthy control group(1) } \\
\text { Group of asymptomatic carriers } \\
\text { of hepatitis B virus(2) }\end{array}$ & 50 & $2.04 \pm 1.01$ & $1.22 \pm 0.50$ \\
Viral hepatitis group(3) & 58 & $8.06 \pm 10.13$ & $1.21 \pm 1.89$ \\
Liver cirrhosis group(4) & 17 & $97.24 \pm 128.18$ & $8.51 \pm 10.71$ \\
Primary liver cancer group(5) & 49 & $80.17 \pm 80.71$ & $8.30 \pm 8.59$ \\
Other hepatobiliary diseases group(6) & 50 & $54.03 \pm 80.34$ & $5.88 \pm 7.89$ \\
\hline
\end{tabular}


Table 2. Comparison of CG and TBA concentration results in each group.

\begin{tabular}{ccccc}
\hline \multirow{2}{*}{ Comparison group } & The comparison of CG concentration & The comparison of TBA concentration \\
\cline { 2 - 4 } & $\mathrm{t}$ value & $\mathrm{p}$ value & $\mathrm{t}$ value & $\mathrm{p}$ value \\
\hline (1)-(2) & 4.5000 & 0.0000 & 0.0388 & 0.4846 \\
(1)-(3) & 3.0622 & 0.0037 & 2.8054 & 0.0063 \\
(1)-(4) & 6.7757 & 0.0000 & 5.7599 & 0.0000 \\
(1)-(5) & 4.5755 & 0.0000 & 4.1680 & 0.0000 \\
(1)-(6) & 3.3514 & 0.0006 & 2.8110 & 0.0030 \\
(2)-(3) & 2.8660 & 0.0056 & 2.7976 & 0.0065 \\
\hline (2)-(4) & 6.2129 & 0.0000 & 5.6630 & 0.0000 \\
(2)-(5) & 4.0186 & 0.0001 & 4.0855 & 0.0000 \\
(2)-(6) & 2.9293 & 0.0021 & 2.7904 & 0.0031 \\
(3)-(4) & 0.5148 & 0.3062 & 0.0814 & 0.4679 \\
(3)-(5) & 1.3055 & 0.1033 & 1.0805 & 0.1458 \\
(3)-(6) & 1.4864 & 0.0754 & 0.7338 & 0.2343 \\
(4)-(5) & 1.6149 & 0.0548 & 1.4603 & 0.0737 \\
(4)-(6) & 1.5474 & 0.0625 & 1.0538 & 0.1473 \\
(5)-(6) & 0.1514 & 0.4400 & 0.0925 & 0.4632 \\
\hline & & & & \\
\hline
\end{tabular}

Serum cholyglycine (CG) is one of the conjugated cholic acids formed by the combination of cholic acid and glycine $[10,11]$. In liver cells, cholesterol undergoes a complex enzymatic reaction and is converted into primary bile acids; Among them are cholic acid (CA) and chenodeoxycholic acid (CD-CA). There are three hydroxyl groups $(\mathrm{C} 3, \mathrm{C} 7, \mathrm{C} 12)$ on the steroid core of cholic acid, and the hydroxyl group at the end of the side chain is combined with glycine by a peptide bond, and the molecular weight is $462 \mathrm{u}$. The normal metabolic pathway of CG is the entero-hepatic circulation. CG is synthesized by hepatocytes, and is discharged into the gallbladder through the capillary bile duct and bile duct, and enters the duodenum with bile to help food digestion. $95 \%$ of bile acids are reabsorbed in the terminal ileum and returned to the liver through the portal vein, where they are taken up and reused by hepatocytes. It mainly exists in the form of protein binding in serum, and the total amount of overflow into the systemic circulation is less than $1 \%$. Under normal circumstances, the content of bile acid in peripheral blood is very small, and the serum CG concentration of normal adults is stable at a low level regardless of fasting or after meals. When hepatocytes are damaged, the ability of hepatocytes to absorb CG decreases, resulting in the increase of CG content in blood; when bile is stagnant, liver excretion of bile acid is hindered, and CG content in reflux blood circulation increases, which also increases blood CG content. Therefore, the determination of serum glycocholic acid (SCG) by RIA (radioimmunoassay) method is one of the sensitive indicators for evaluating the function of hepatocytes and the circulation of hepatobiliary substances. Cholyglycine is the most important bile acid component in serum in late pregnancy. When hepatocytes are damaged, the ability of hepatocytes to take up CG decreases, resulting in an increase in the level of CG in blood. When bile is 
stagnant, the liver excretion of bile acid is hindered, and the CG content in the reflux blood circulation increases, which also increases the blood CG content. The content of serum glycocholic acid in normal people is $1.3 \pm 0.8 \mathrm{mg} / \mathrm{l}$, ranging from 0.4 to $2.98 \mathrm{mg} / \mathrm{l}$; the lower limit of hepatitis diagnosis is $<3.18 \mathrm{mg} / \mathrm{l}$. Total bile acid (TBA) is a group of metabolites of cholesterol in liver decomposition and entero-hepatic circulation; TBA is the final product of cholesterol catabolism in liver and is closely related to the absorption, metabolism and regulation of cholesterol $[12,13]$. The total bile acids of human body are divided into primary bile acids and secondary bile acids; Primary bile acids take cholesterol as raw material and participate in the digestion and absorption of fat. After entering the duodenum through the biliary system, it is hydrolyzed to produce secondary bile acids under the action of intestinal bacteria; when the liver cell is damaged or blocked inside and outside the liver, the metabolism of bile acid is impaired and flows back into the blood, and the concentration of serum total bile acid increases; therefore, the change of total bile acid level can sensitively reflect liver function. Serum total bile acid is a sensitive diagnostic index for liver parenchymal injury and digestive system diseases; Total bile acid (TBA) can reflect the excretion function of liver more specifically, and the increase of tBA can be caused by the pathological changes of liver cells or the disorder of enteric-liver circulation. Serum total bile acid increase: it can be seen in various acute and chronic hepatitis, hepatitis B carriers or alcoholic hepatitis; TBA is more sensitive than all other liver function tests to detect mild liver disease, and can also be seen in most extrahepatic bile duct obstruction and intrahepatic cholestatic diseases, liver cirrhosis, obstructive jaundice, etc.

The results of this study showed that, by statistical analysis, serum CG concentration in the viral hepatitis group, liver cirrhosis group, primary liver cancer group and other hepatobiliary disease groups were significantly higher than those in asymptomatic carriers of hepatitis B virus and healthy controls, and the differences were statistically significant, $\mathrm{p}<0.05$. While, the serum CG concentration in asymptomatic carriers of hepatitis B virus was similar to that in healthy controls, and there was no significant difference between them ( $p>0.05)$. There was no significant difference in the concentration of CG among viral hepatitis group, liver cirrhosis group, primary liver cancer group and other hepatobiliary diseases group, $p>$ 0.05. Serum TBA levels in asymptomatic carriers of hepatitis B virus, viral hepatitis group, liver cirrhosis group, primary liver cancer group and other hepatobiliary disease groups were significantly higher than those in the healthy control group, the differences were all statistically significant, $\mathrm{p}<0.05$. There was no significant difference in the levels of TBA concentration among the asymptomatic carriers of hepatitis B virus, viral hepatitis group, liver cirrhosis group, primary liver cancer group and other hepatobiliary system disease groups, all $\mathrm{p}>0.05$. This suggests that the expression of serum CG and TBA can not only detect liver lesions, but also distinguish different liver lesions. The positive rate of CG combined with TBA detection in patients with hepatobiliary diseases was significantly higher than that of single CG index detection, and the sensitivity and accuracy of CG combined with TBA detection were significantly improved, indicating that CG combined with TBA detection has certain complementarity in the diagnosis of hepatobiliary diseases.

\section{CONCLUSION}

Serum CG expression can not only detect liver lesions, but also distinguish different liver lesions. The positive rate of CG combined with TBA detection in patients with hepatobiliary diseases is significantly higher than that of single CG index detection.CG combined with TBA detection can significantly improve the sensitivity and accuracy of the diagnosis of hepatobiliary diseases, and is worthy of popularization and application.

\section{LIMITATIONS OF THE STUDY}

All the subjects in this study were from inpatients in our hospital and the health check-up population in the physical examination center. There are certain geographical differences and cannot fully represent the situation in other regions, so it has certain limitations. 


\section{ACKNOWLEDGEMENTS}

This project was approved and received strong support from the functional departments of the hospital and the help of colleagues. I would like to express my heartfelt thanks to them for their support and help! Wish them good health, smooth work and all the best!

\section{FUND PROJECT}

The clinical application value of the combined detection of CG and TBA in the differential diagnosis of hepatobiliary diseases (NO. 2019YFF0216502) which is a sub-project of the National Key Research and Development Program of "Research and Application of Common Technology of National Quality Foundation" of biochemical diagnostic reagents related to senile diseases.

\section{CONFLICTS OF INTEREST}

The authors declare no conflicts of interest regarding the publication of this paper.

\section{REFERENCES}

1. Han, W., Li, H.Q., Li, J.J., et al. (2021) Application Value of CG, TBA Combined with AFP and PIVKA-II Detection in the Diagnosis of HBV Infection-Related Liver Diseases. Marker Immunoassay and Clinical, 28, 1584-1589.

2. Wang, X.Y., Zhang, T. and Jing, C.B. (2020) Meta-Analysis of Serum Total Bile Acid and Glycocholic Acid Levels in the Diagnosis of Intrahepatic Cholestasis of Pregnancy. Journal of Modern Laboratory Medicine, 35, 73-76.

3. Liang, Y.L., Xie, X.L., Ren, Y.P., et al. (2019) Application of Glycocholic Acid in Common Liver Diseases. Chinese Physician Journal, 21, 387-391.

4. Xie, M.H., Luo, Y.C. and Zheng, H.L. (2010) Clinical Significance of Serum Total Bile Acid and Hepatic Bile Acid Determination in Patients with Liver Disease. Chinese National Folk Medicine, 19, 135-135.

5. Zhang, Y.T., Song, Y.H., Huang, H.F., et al. (2017) Clinical Practice of Glycocholic Acid Detection and Comparison with Other Liver Function Indicators. International Journal of Laboratory Medicine, 38, 680-682.

6. Guo, N.L., Du, S.X. and Yang, Y.W. (2001) Clinical Significance of Determination of Total Bile Acid and Glycocholic Acid in Neonatal Hyperbilirubinemia. Hebei Medicine, 23, 209-210.

7. Hino, K., Yamamoto, S., Yamashita, S., et al. (1981) A Comparative Study of Serum Bile Acid Levels (TBA, CG) in Liver Disease-Difference between Fasting and UDCA Oral Loading (Author's Transl). Nihon Shokakibyo Gakkai zasshi (The Japanese Journal of Gastro-Enterology), 78, 2197.

8. Kazunari, H. (1981) A Comparative Study of Serum Bile Acid Levels (Tba, Cg) in Liver Disease-Difference between Fasting and Udca Oral Loading. Nippon Shokakibyo Gakkai Zasshi, 78, 2197-2197.

9. Zhang, Q.Q., Jin, L. and Gong, H. (2011) Clinical Significance of Serum CG, ALT and TBA Detection in Pregnant Women with Hepatitis B Infection. Journal of Radioimmunology, 24, 537-538.

10. Kang, J.H. Tan, C.K. and Zhang, J.W. (2018) The Diagnostic Significance of Glycocholic Acid in Chronic Liver Disease. Journal of Hubei University of Traditional Chinese Medicine, 20, 102-103.

11. Liu, X.M. and Gan, F.S. (2021) The Value of Serum Glycocholic Acid in the Diagnosis of Liver Disease. Chinese Contemporary Medicine, 28, 171-174. https://doi.org/10.1155/2021/6698085

12. Li, H., Hao, X.Y. and Zhao, L.F. (2006) The Relationship between Serum Total Bile Acid Level and Tension Cholecystitis in Patients with Chronic Hepatitis. Journal of Shanxi Medical University, 37, 190-191. 
13. Liu, F.H., Wang, L.J., Zhou, Y.H., et al. (2017) Exploring the Application Value of Combined Detection of 15 Biochemical Indicators in the Treatment of Malignant Liver Tumors after the First Treatment. Laboratory Medicine, 5, 353-360. 